\title{
NLP Data Cleansing Based on Linguistic Ontology Constraints
}

\author{
Dimitris Kontokostas ${ }^{1}$, Martin Brümmer ${ }^{1}$, Sebastian Hellmann ${ }^{1}$, Jens \\ Lehmann $^{1}$, and Lazaros Ioannidis ${ }^{2}$ \\ 1 Universität Leipzig, Institut für Informatik, AKSW, http://aksw.org \\ \{lastname\}@informatik.uni-leipzig.de \\ 2 Aristotle University of Thessaloniki, Medical Physics Laboratory \\ lioannid@math.auth.gr
}

\begin{abstract}
Linked Data comprises of an unprecedented volume of structured data on the Web and is adopted from an increasing number of domains. However, the varying quality of published data forms a barrier for further adoption, especially for Linked Data consumers. In this paper, we extend a previously developed methodology of Linked Data quality assessment, which is inspired by test-driven software development. Specifically, we enrich it with ontological support and different levels of result reporting and describe how the method is applied in the Natural Language Processing (NLP) area. NLP is - compared to other domains, such as biology - a late Linked Data adopter. However, it has seen a steep rise of activity in the creation of data and ontologies. NLP data quality assessment has become an important need for NLP datasets. In our study, we analysed 11 datasets using the lemon and NIF vocabularies in 277 test cases and point out common quality issues.
\end{abstract}

Keywords: \#eswc2014Kontokostas, Linked Data, NLP, data quality

\section{Introduction}

Linked Data (LD) comprises of an unprecedented volume of structured data on the Web and is adopted from an increasing number of domains. However, the varying quality of the published data forms a barrier in further adoption, especially for Linked Data consumers.

Natural Language Processing (NLP) is - compared to other domains, such as Biology - a late LD adopter with a steep rise of activity in the creation of vocabularies, ontologies and data publishing. A plethora of workshops and conferences such as LDL http://1d12014.org/, WoLE http://wole2013.eurecom.fr, LREC http: //1rec2014.1rec-conf.org, MLODE http://sabre2012.infai.org/mlode, NLP\&DBpedia http: //nlp-dbpedia2013.blogs.aksw.org/program/) motivate researchers to adopt Linked Data and RDF/OWL and convert traditional data formats such as XML and relational databases. Although guidelines and best practices for this conversion exist, developers from NLP are often unfamiliar with them, resulting in low quality and inoperable data. In this paper, we address the subsequently arising need for data quality assessment of those NLP datasets. 


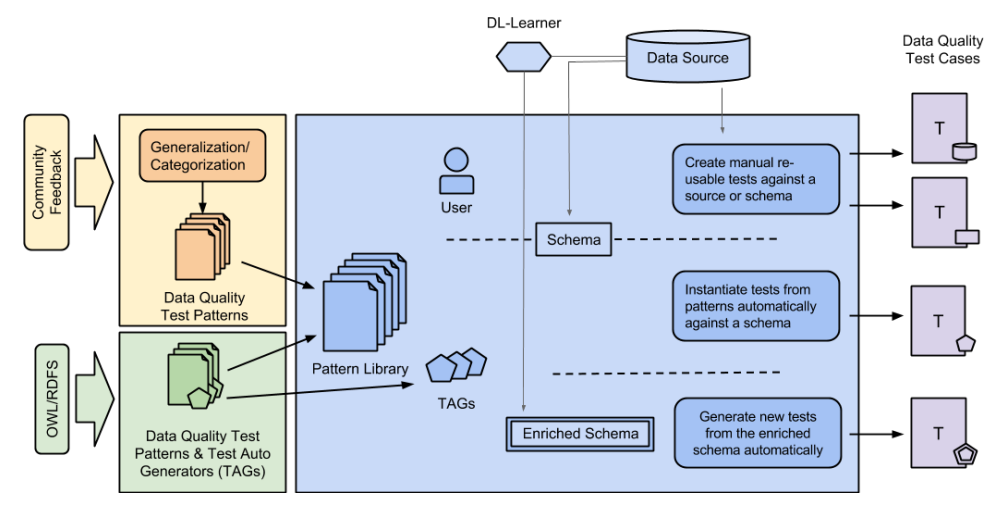

Fig. 1. Flowchart showing the test-driven data quality methodology. The left part displays the input sources of our pattern library. In the middle part the different ways of pattern instantiation are shown which lead to the Data Quality Test Cases on the right.

We extended a recently introduced test-driven data quality methodology 12 inspired by tests in software engineering. In its introduction, the methodology in 12 focused on two approaches: (1) automatically-generated test cases which were derived from the OWL/RDFS schema of the ontologies and (2) test cases adhering to patterns from a pattern library. These two approaches were evaluated on popular ontologies and data sets such as FOAF or the DBpedia Ontology and it was shown that the methodology is well suited for horizontal, multi-domain data quality assessment, i.e. massive detection of errors for five large-scale LOD data sets as well as on 291 vocabularies, independent of their domain or their purpose. In this paper, we will briefly introduce the methodology in Section 2 including a comparison of our methodology to OWL reasoning. The Test Driven Data Engineering Ontology is described in Section 3. Using the ontology, we can annotate test cases and provide support for different levels of result reporting allowing to give feedback to developers when running these tests and ultimately improving data quality.

Additionally, we show progress in implementing domain-specific validation by quickly improving existing validation provided by ontology maintainers. We specifically analysed datasets for two emerging domain ontologies, the lemon model [13] and the NIF 2.0 Core Ontology [10] in Section 4 and evaluated 11 datasets in Section 5 .

\section{Overview of Test-Driven Data Assessment Methodology}

In this section we introduce basic notions of our methodology. A thorough description of test-driven quality assessment methodology can be found in 12

Data Quality Test Pattern (DQTP). A data quality test pattern is a SPARQL query template with variable placeholders. Possible types of the pattern 
variables are IRIs, literals, operators, datatype values (e.g. integers) and regular expressions. Using \%\%v\% as syntax for placeholders, an example DQTP is:

SELECT ?s WHERE \{ ?s $\% \%$ P $1 \% \%$ ?v 1 ? ? $\%$ P $2 \% \%$

FILTER ( ?v1 \% ?

This DQTP can be used for testing whether a value comparison of two properties $P 1$ and $P 2$ holds with respect to an operator $O P$. DQTPs represent abstract patterns, which can be further refined into concrete data quality test cases using test pattern bindings.

Test Pattern Binding. Test pattern bindings are valid DQTP variable replacements.

Data Quality Test Case. Applying a pattern binding to a DQTP results in an executable SPARQL query. Each result of the query is considered to be a violation of a test case. A test case may have four different results: success (empty result), violation (results are returned), timeout (test is marked for further inspection) and error (something prevented the query execution). An example test pattern binding and resulting data quality test case is $3^{3}$

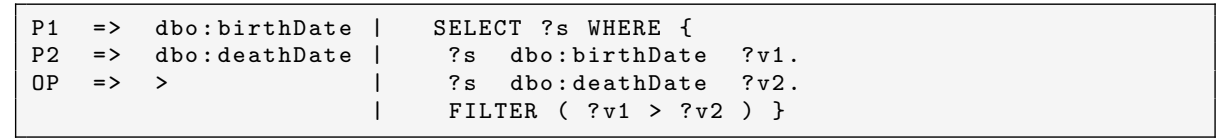

Test Auto Generator (TAG). A Test Auto Generator reuses the RDFS and OWL modelling of a knowledge base to verify data quality. In particular, a TAG, based on a DQTP, takes a schema as input and returns test cases. TAGs consist of a detection and an execution part. The detection part is a query against a schema and for every result of a detection query, a test case is instantiated from the respective pattern, for instance:

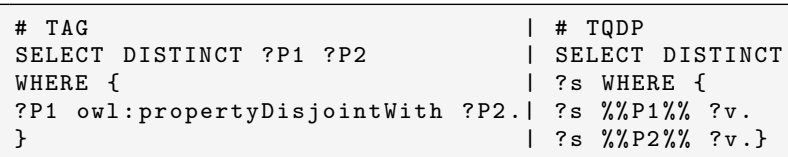

Additionally, we devise the notion of RDF test case coverage based on a combination of six individual coverage metrics 12 .

The test-driven data quality methodology is illustrated in Figure 1 As shown in the figure, there are two major sources for the creation of tests. One source is stakeholder feedback from everyone involved in the usage of a dataset and the other source is the already existing RDFS/OWL schema of a dataset. Based on this, there are several ways to create tests:

1. Manually create test cases: Test cases specific to a certain dataset or schema can be written manually. This can be guided choosing suitable DQTPs of

\footnotetext{
${ }^{3}$ We use http://prefix.cc to resolve all name spaces and prefixes. A full list can be found at http://prefix.cc/popular/all
} 
our pattern library. Tests that refer to the schema of a common vocabulary can become part of a central library to facilitate later reuse.

2. Reusing tests based on common vocabularies: Naturally, a major goal in the Semantic Web is to reuse existing vocabularies instead of creating new ones. We detect the used vocabularies in a dataset, which allows to re-use tests from a test pattern library.

3. Using RDFS/OWL constraints directly: As previously explained, tests can be automatically created via TAGs in this case.

4. Enriching the RDFS/OWL constraints: Since many datasets provide only limited schema information, we perform automatic schema enrichment as recently researched in [4]. Those schema enrichment methods can take an RDF dataset or a SPARQL endpoint as input and automatically suggest schema axioms with a certain confidence value by analysing the dataset. In our methodology, this is used to create further tests via TAGs.

The RDFUnit Suite ${ }^{4} 5^{5}$ implements the test driven data assessment methodology The methodology is implemented in a Java component and released as open source under the Apache licence.

Relation Between SPARQL Test Cases and OWL Reasoning. SPARQL test cases can detect a subset of common validation errors detectable by a sound and complete OWL reasoner. However, this is limited by a) the reasoning support offered by the used SPARQL endpoint and b) the limitations of the OWL-toSPARQL translation. On the other hand, SPARQL test cases can find validation errors that are not expressible in OWL, but within the expressivity of SPARQL (see 1] for more details and a proof that SPARQL 1.0 has the same expressive power as relational algebra under bag semantics). This includes aggregates, property paths, filter expressions etc. Please note that for scalability reasons full OWL reasoning is often not feasible on large datasets. Furthermore, many datasets are already deployed and easy to access via SPARQL endpoints. Additionally, the Data Quality Test Pattern (DQTP) library may arguably provide a more user friendly approach for building validation rules compared to modelling OWL axioms. However, the predefined DQTP library has some limitations as well, in particular a) it requires familiarity with the library in order to choose the correct DQTP and 2) custom validations cannot always correspond to an existing DQTP and manual SPARQL test cases are required.

\section{Test Driven Data Engineering Ontology}

The Test Driven Data Assessment methodology is implemented using RDF as input and output and complies with our accompanied ontology ${ }_{6}^{6}$ The ontology additionally serves as a self-validation layer for the application input (test-cases, DQTPs and TAGs) and output (validation results). The ontology consists of 20

\footnotetext{
4 https://github.com/AKSW/RDFUnit

5 http://RDFUnit.aksw.org

6 http://RDFUnit.aksw.org/ns/core\#
} 


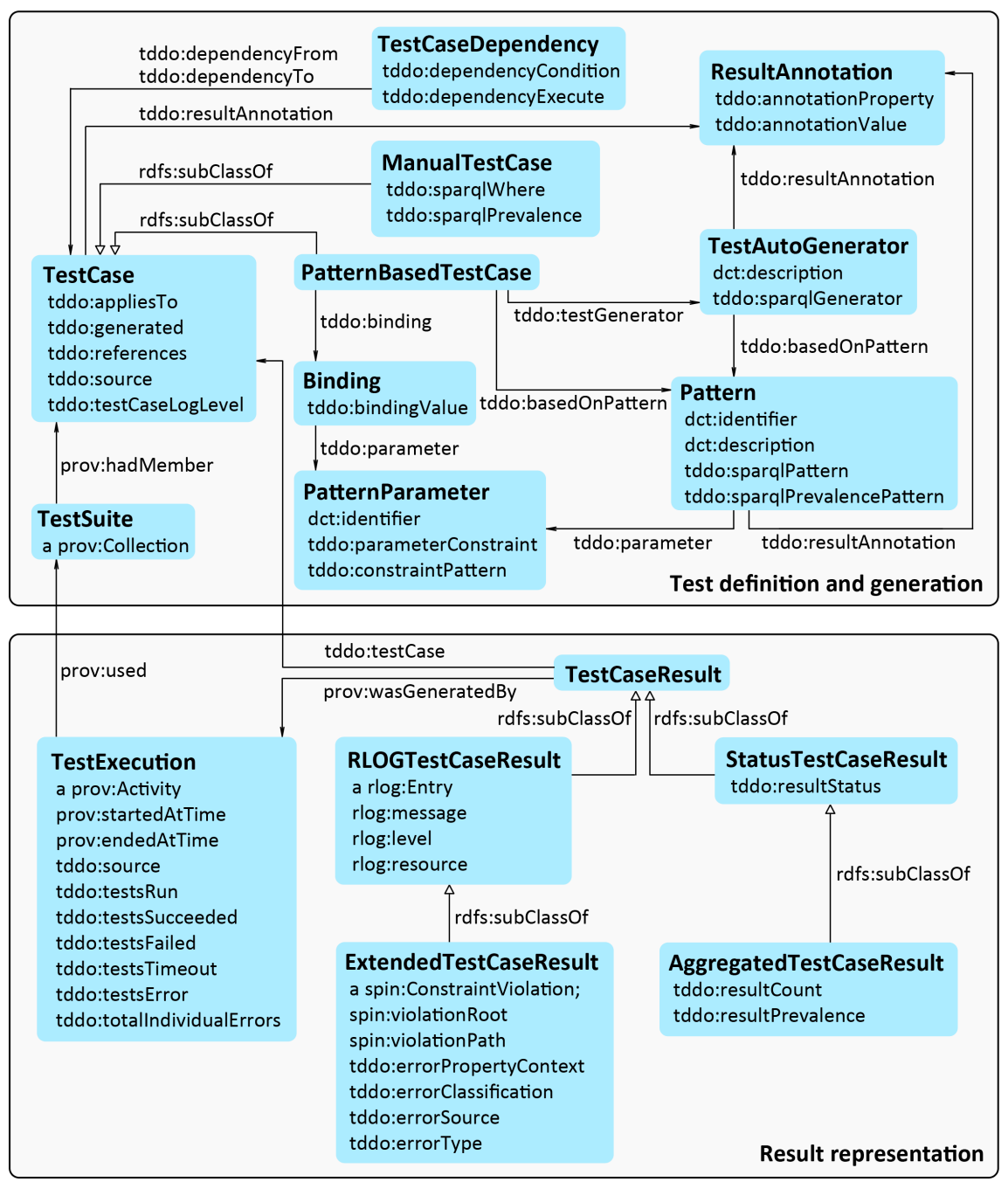

Prefix prov: http://www.w3.org/ns/prov\#

Prefix spin: http://spinrdf.org/spin\#

Prefix dct: $\mathrm{http}: / /$ purl.org/dc/terms/

Prefix tddo: http://databugger.aksw.org/ns/core\#

Prefix rlog: http://persistence.uni-leipzig.org/nlp2rdf/ontologies/rlog\#

Fig. 2. Class dependencies for the test driven data engineering ontology.

classes and 36 properties and reuses the PROV [2, RLOG ${ }^{7}$ and spir ${ }^{8}$ ontologies. As depicted in Figure 2, the ontology is centered around two concepts, the test case definition and generation and the result representation.

7 http://persistence.uni-leipzig.org/nlp2rdf/ontologies/rlog\#

8 http://spinrdf.org 
Test case definition and generation. We encapsulate a list of test cases in a TestSuite, a subclass of prov:Collection that enumerates the contained test cases with prov: hadMember. The class TestCase describes an abstract test case. For each test case, we provide provenance with the following properties:

- :appliesTo to denote whether the test case applies to a schema, a dataset or an application.

- : source, the URI of the schema, dataset or application.

- :generated on how the test case was created (automatic or manually).

- :references a list of URIs a test case uses for validation.

- :testCaseLogLevel an rlog:Level this test case is associated with. In accordance to software development, the available log levels are: TRACE, DEBUG, INFO, WARN, ERROR and FATAL.

Additionally, each TestCase is associated with two SPARQL queries, a query for the constraint violations and a query for the prevalence of the violations. The prevalence query is optional because it cannot be computed in all cases.

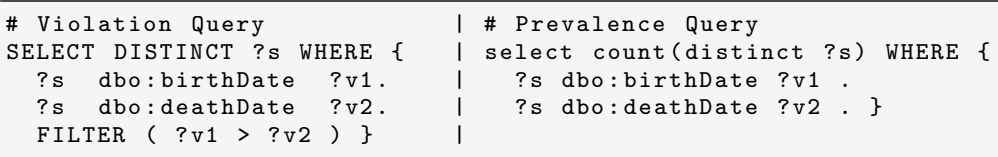

Concrete instantiations of a TestCase are the ManualTestCase and the PatternBasedTestCase classes. In the former, the tester defines the SPARQL queries manually while the in the latter she provides Bindings for a Pattern. Additionally, the ontology allows the definition of dependencies between test cases. For example if test case $A$ fails, do not execute test case B. This is achieved with the TestCaseDependency class where : dependencyFrom and :dependencyTo define the dependent test cases, :dependencyCondition is the status result that triggers an execute or don't execute (:dependencyExecute) for the dependant test case.

A Pattern is identified and described with the dct:identifier and dct:description properties. The : sparqlPattern and :sparqlPrevalencePattern properties hold the respective SPARQL queries with placeholder for replacement. For each placeholder a PatternParameter is defined and connected to the pattern with the : parameter property.

PatternParameters are described with a dct:identifier and two restriction properties: the : parameterConstraint to restrict the type of a parameter to Operator, Resource, Property or Class and the optional : constraintPattern for a regular expression constraint on the parameter values.

Bindings link to a PatternParameter and a value through the : parameter and : bindingValue properties respectively. PatternBasedTestCases are associated with Bindings through the : binding property.

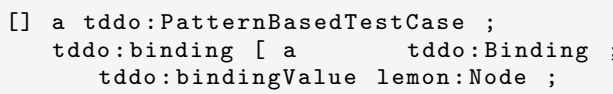


A PatternBasedTestCase can be automatically instantiated through a TestAutoGenerator. Generators hold a dct:description, a sparql query (:generatorSparql) and a link to a pattern (: basedOnPattern).

Result representation. For the result representation we reuse the PROV Ontology. The TestExecution class is a subclass of prov: Activity that executes a TestSuite (prov:used) against a : source and generates a number of TestCaseResults. Additional properties of the TestExecution class are prov:startedAtTime and prov: endedAtTime as well as aggregated execution statistics like: :testsRun, :testsSucceeded, :testsFailed, :testsTimeout, :testsError and :totalIndividualErrors.

The ontology supports four levels or result reporting, two for report on the test case level and two for individual error reporting. All result types are subclasses of the TestCaseResult class and for provenance we link to a TestCase with :testCase and a TestExecution with prov:wasGeneratedBy properties. The StatusTestCaseResult class contains a single :resultStatus that can be one of Success, Fail, Timeout and Error. The AggregatedTestCaseResult class adds up to the StatusTestCaseResult class by providing an aggregated view on the individual errors of a test case with the properties :resultCount and :resultPrevalence.

For the individual error reporting the RLOGTestCaseResult generates logging messages through the RLog ontology. For every violation, we report the erroneous resource ( $\mathrm{rlog}$ : resource), a message ( $\mathrm{rlog}$ :message) and a logging level (rlog:level). The logging level is retrieved from the TestCase.

The ExtendedTestCaseResult class extends RLOGTestCaseResult by providing additional properties for error debugging by reusing the spin ontology. In detail, an ExtendedTestCaseResult is a subclass of spin:ConstraintViolation and may have the following properties:

- spin:violationRoot: the erroneous resource.

- spin:violationPath: the property of the resource that the error occurs.

- :errorPropertyContext: lists additional properties that may provide a better context for fixing the error. For example, in the dbo: birthDate before a dbo: deathDate case, dbo:birthDate can be the spin:violationPath and $\mathrm{dbo}$ : deathDate the :errorPropertyContext.

- :errorClassification: is a sub-property of dct:subject that points to a SKOS error classification category.

- :errorSource: is a sub-property of dct:subject that points to a SKOS error source category. Example values can be data parsing, data publishing, mapping, pre processing, post processing, etc.

- :errorType: is a sub-property of dct:subject and that points to a SKOS error type category on the triple level. Example values can be: missing property, redundant property, inaccurate property.

The extended error annotation is generated through the ResultAnnotation class that is attached to a TestCase through the :resultAnnotation property. A ResultAnnotation must contain an :annotationProperty linking to one of the allowed ExtendedTestCaseResult properties and an appropriate value for : annotationValue. For the schema-based automatic test case generation some 


\begin{tabular}{|l|r|r|r|r|r|r|r|r|}
\hline & Total & Domain & Range & Datatype & Card. & Disj. & Func. & I. Func. \\
\hline Lemon & 172 & 40 & 34 & 1 & 29 & 64 & 3 & 1 \\
\hline NIF & 86 & 42 & 24 & 4 & & 6 & 10 & \\
\hline
\end{tabular}

Table 1. Number of automatically generated test cases per ontology. We provide the total number of test cases as well as separated per rdfs domain and range, literal datatype, OWL cardinality (min, max, exact), property \& class disjointness, functional and inverse functional constraints.

of the annotation may be known only on the Pattern level and other on the TestAutoGenerator level. Thus, ResultAnnotations are allowed in both classes and the error annotation are added up on the test case generation.

Finally, we provide :testSuite, an ontology annotation property, that links an ontology to an appropriate TestSuite for data validation purposes.

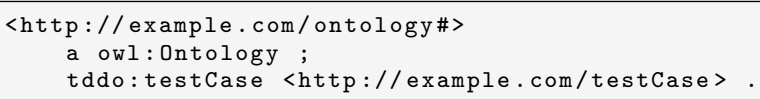

\section{Test Case Implementation for Linguistic Ontologies}

In this section, we will discuss the employment of RDFUnit for lemon and NIF, especially with regard to these questions: (1) What is the coverage of the automatically generated tests, what are their limitations. (2) Where is it feasible to use the predefined patterns from the pattern library [12]? Are there test cases that are too complex and need manual creation by an expert? (3) Which test cases can not be expressed at all as they are not expressible via SPARQL?

By running the existing RDFUnit Test Auto Generators (TAG) on the lemon and NIF ontologies we automatically generated 172 test cases for lemon and 86 test cases for NIF (cf. Table 1). Both ontologies are of similar size: NIF contains 19 classes and 46 properties while lemon 23 classes and 55 properties. The number of increased test cases in lemon results from the higher amount of defined cardinality and disjointness restrictions. The RDFUnit Suite, at the time of writing, does not provide full OWL coverage and thus, complex owl:Restrictions cannot be handled yet. In the frame of the examined ontologies, RDFUnit did not produce test cases for unions of (owl:unionOf) restrictions such as multiple cardinalities for lemon:LexicalSense and lemon:LemonElement or restrictions with owl:allValuesFrom, owl:someValuesFrom and owl:hasSelf for NIF.

Both NIF and lemon have defined semantic constraints that can not be captured in OWL and are too complex for the above-mentioned pattern library. In particular, NIF and lemon use natural language text in the rdfs:comment properties as well as their documentations and specification documents. 
For lemon, the maintainers implemented a Python validator 9 , which enables us to directly compare our efforts to a software validator. For NIF there was an early prototype of RDFUnit that used only manual SPARQL test cases.

\subsection{Lemon}

According to Table 1, test cases for rdfs:domain and rdfs:range restrictions are the largest group, at $43.8 \%$, followed by tests for disjointness (37.4\%) and cardinality restrictions (18.8\%). The existing lemon validator contains 24 test cases for some structural criteria of the lemon ontology. 14 of these tests are natively covered by the existing RDFUnit TAGs. Out of the 10 remaining cases, four where on warning and info level, based on recommendations from the ontology's guidelines. They are thus not explicitly stated in OWL, because they don't constitute logical errors and can not be covered by automatic test generation. Of the six remaining errors, two where expressed via owl: union0f and two could not be expressed by the ontology's author because OWL is not able to express them. Additionally, the lemon validator reported undeclared properties under the lemon namespace. Although this test case can be expressed in SPARQL, it was not implemented at the time of writing.

The last error case not covered was due to an error in the ontology itself. Lemon defines that every instance of lemon: LexicalEntry may have a maximum of one lemon: canonicalForm property. Yet, the validator fails if the instance has no lemon: canonicalForm, thus suggesting that instead of the owl : maxCardinality, a owl: cardinality restriction was intended in this case. These kind of semantic subtleties are usually very hard to detect in the complex domain of ontology engineering. It shows that the intensive engagement necessary to write the test cases already serves to debug the ontologies underlying the datasets. This extends the test-driven approach to the ontology development, apart from the quality assessment.

These test cases could directly be translated into SPARQL queries for testing with RDFUnit. For example, it is suggested that a lemon:LexicalEntry should contain an rdfs:label. As there is no possibility to express these optional constraints in OWL, this test case was added manually to log matching resources as an info-level notice.

Beyond the implementation of the lemon validator as test cases, some additional test cases were added to test for semantic correctness or properties that could be added. For example, the lemon: narrower relation, which denotes that one sense of a word is narrower than the other, must never be symmetric or contain cycles.

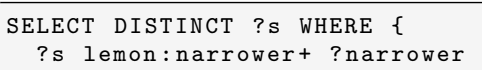

$9 \longdiv { \text { https://github.com/jmccrae/lemon-model.net/blob/master/validator/lemon-validator.py } }$ 
Similarly, if one resource is lemon:narrower to another resource, the inverse relationship (lemon:broader) should exist in the database.

From the total of ten manual test cases that were defined for lemon, five were described as PatternBasedTestCases, using the existing pattern library, and five as ManualTestCases using custom SPARQL queries. However, for brevity we described the test case with the final SPARQL queries.

\subsection{NIF}

Almost 50\% of automated NIF test cases were for rdfs : domain constraints, $27 \%$ for rdfs : range, $11 \%$ for owl:FunctionalPropery restrictions, $7 \%$ for disjointness and $5 \%$ for proper datatype usage. The early prototype of RDFUnit that is used as the NIF validator did not cover any schema constraints and consists of 10 test cases. There exists one test case on the warning level that reports classes of the old namespace.

Other manual test cases include the following restrictions:

- An occurrence of nif : beginIndex inside a nif: Context must be equal to zero $(0)$.

- The length of nif:isString inside a nif: Context must be equal to nif: endIndex.

- A nif : anchorOf string must match the substring of the nif:isString from nif : beginIndex to nif : endIndex. For example:

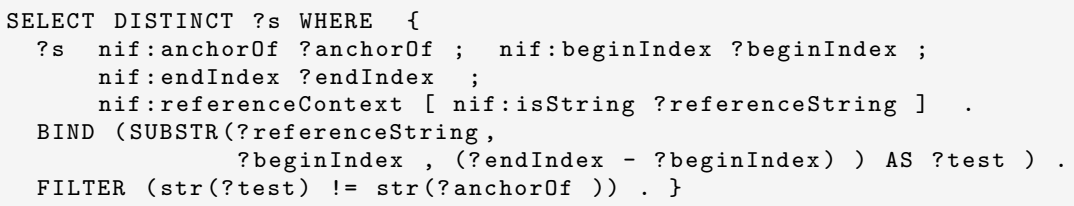

- nif:CString is an abstract class and thus a subclass such as nif: CStringImpl or nif:RFC5147String must be used.

- All instances of nif:CString that are not nif:Context must have a nif: referenceContext property.

- All instances of nif:Context must also be instances of a nif:CString subclass.

- Misspelled rdf : type declarations for class names, for example nif : RFC5147String.

- All instances of nif:CString must have the properties nif:beginIndex and nif: endIndex.

- all nif:Context must have an explicit nif:isString, nif:isString can only occur with nif: Context.

\section{Evaluation}

$1 0 \longdiv { \text { http://lemon-model.net/lexica/uby/WktDE/WktEN.nt.gz } }$ 


\begin{tabular}{|c|c|c|c|}
\hline Name & Description & Ontology & Type \\
\hline \multicolumn{4}{|l|}{ lemon datasets } \\
\hline $\begin{array}{l}\text { LemonUby } \\
\text { Wiktionary EN }{ }^{10}[5]\end{array}$ & $\begin{array}{l}\text { Conversion of the English Wiktionary into } \\
\text { UBY-LMF model }\end{array}$ & $\begin{array}{l}\text { lemon, } \\
\text { UBY-LMF }\end{array}$ & Dictionary \\
\hline $\begin{array}{l}\text { LemonUby } \\
\text { Wiktionary DE }{ }^{11}[5]\end{array}$ & $\begin{array}{l}\text { Conversion of the German Wiktionary into } \\
\text { UBY-LMF model }\end{array}$ & $\begin{array}{l}\text { lemon, } \\
\text { UBY-LMF }\end{array}$ & Dictionary \\
\hline $\begin{array}{l}\text { LemonUby Wordnet } \\
12 \text { [5] }\end{array}$ & $\begin{array}{l}\text { Conversion of the Princeton WordNet } 3.0 \text { into } \\
\text { UBY-LMF model }\end{array}$ & $\begin{array}{l}\text { lemon, } \\
\text { UBY-LMF }\end{array}$ & WordNet \\
\hline $\begin{array}{l}\text { DBpedia Wiktionary } \\
13 \text { [9] }\end{array}$ & $\begin{array}{l}\text { Conversion of the English Wiktionary into } \\
\text { lemon }\end{array}$ & lemon & Dictionary \\
\hline QHL ${ }^{14}[15]$ & $\begin{array}{l}\text { Multilingual translation graph from more than } \\
50 \text { lexicons }\end{array}$ & lemon & Dictionary \\
\hline \multicolumn{4}{|l|}{ NIF datasets } \\
\hline Wikilinks $[15[10]$ & $\begin{array}{l}\text { sample of } 60976 \text { randomly selected phrases } \\
\text { linked to Wikipedia articles }\end{array}$ & NIF & NER \\
\hline $\begin{array}{l}\text { DBpedia Spotlight } \\
{\text { datase }{ }^{16}[18}^{16}\end{array}$ & $\begin{array}{l}58 \text { manually NE annotated natural language } \\
\text { sentences }\end{array}$ & NIF & NER \\
\hline $\begin{array}{l}\text { KORE } 50 \text { evaluation } \\
\text { datase }\left[{ }^{17}[18]\right.\end{array}$ & $\begin{array}{l}50 \mathrm{NE} \text { annotated natural language sentences } \\
\text { from the AIDA corpus }\end{array}$ & NIF & NER \\
\hline News-10 ${ }^{18}[16]$ & 100 manually annotated German news articles & NIF & NER \\
\hline $\mathrm{RSS}^{-50}{ }^{19} 16$ & $\begin{array}{l}500 \text { manually annotated sentences from } 1,457 \\
\text { RSS feeds }\end{array}$ & NIF & NER \\
\hline $\begin{array}{lll}\text { Reuters-12 } & 20 & 16]\end{array}$ & 128 news articles manually curated & NIF & NER \\
\hline
\end{tabular}

Table 2. Tested datasets

For evaluation purposes we gathered a representative sample of lemon and NIF datasets in Table 2. We loaded all the datasets in an open-source edition of

11 http://lemon-model.net/lexica/uby/WktDE/WktDE.nt.gz

12 http://lemon-model.net/lexica/uby/wn/wn.nt.gz

13 http://downloads.dbpedia.org/wiktionary/dumps/en/wiktionary_en_2013-09-17_dump-20130726.

ttl.bz2

14 http://linked-data.org/datasets/qhl.ttl.zip

15 http://mlode.nlp2rdf.org/datasets/wikilinks-sample.ttl.tar.gz

16 http://www.yovisto.com/labs/ner-benchmarks/data/dbpedia-spotlight-nif.ttl

17 http://www.yovisto.com/labs/ner-benchmarks/data/kore50-nif.ttl

18 https://raw.github.com/AKSW/n3-collection/master/News-100.ttl

19 https://raw.github.com/AKSW/n3-collection/master/RSS-500.ttl

20 https://raw.github.com/AKSW/n3-collection/master/Reuters-128.ttl 


\begin{tabular}{|l||r||r|r|r|r||r|r|r|r|}
\hline & Size & SC & FL & TO & ER & AErrors & MErrors & MWarn & MInfo \\
\hline WiktDBp & $60 \mathrm{M}$ & 177 & 5 & - & - & 3.746 .103 & 7.521 .791 & -3.582 .837 \\
\hline WktEN & $8 \mathrm{M}$ & 168 & 14 & - & - & 752.018 & 394.766 & - & 633.270 \\
\hline WktDE & $2 \mathrm{M}$ & 170 & 12 & - & - & 273.109 & 66.268 & - & 155.598 \\
\hline Wordnet & $4 \mathrm{M}$ & 166 & 16 & - & - & 257.228 & 36 & - & 257.204 \\
\hline QHL & $3 \mathrm{M}$ & 170 & 11 & - & 1 & 433.118 & 538.933 & - & 538.016 \\
\hline Wikilinks & $0.6 \mathrm{M}$ & 91 & 4 & - & 1 & 141.528 & 21.246 & - & - \\
\hline News-100 & $13 \mathrm{~K}$ & 91 & 2 & - & 3 & 3.510 & - & - & - \\
\hline RSS-500 & $10 \mathrm{~K}$ & 91 & 2 & - & 3 & 3.000 & - & - & - \\
\hline Reuters-128 & $7 \mathrm{~K}$ & 91 & 2 & - & 3 & 2.016 & - & - & - \\
\hline Spotlight & $3 \mathrm{~K}$ & 92 & 3 & - & 1 & 662 & 68 & - & - \\
\hline KORE50 & $2 \mathrm{~K}$ & 89 & 6 & - & 1 & 301 & 55 & - & - \\
\hline
\end{tabular}

Table 3. Overview of the NLP datasets test execution. For every dataset, we provide the size in triples count, the number of test cases that were successful, failed, timed-out and did not complete due to an error. Additionally, we mention the total the number of the individual violations from automated test cases along with errors, warnings and infos from manual test cases.

Virtuoso server (version 7.0) ${ }^{21}$ and ran RDFUnit for each one of them. The results of the dataset evaluation are provided in Table 3.

Looking at the results of Table 3 we observe that manual test cases can be of equal importance to the schema restrictions. Additionally we notice that the lemon-based datasets were more erroneous than the NIF-based datasets. This may be attributed to the following reasons:

- the NIF datasets were smaller in size and, thus, better curated.

- the DBpedia Wiktionary datasets is derived from a crowd-sourced source, which makes it more prone to errors.

- the lemon ontology is stricter than the NIF ontology.

- 16] already used the early prototype of RDFUnit and fixed all data errors found by manual test cases.

All lemon datasets failed the info level test case that required at least one and unique lemon:language in a lemon:LexicalEntry. The existence of a lemon:subsense or exactly one lemon:reference also failed in all datasets with a high number of violations, except Wordnet that had only 33. Additionally, all datasets had a high number of violation on the owl:minCardinality of 1 constraint of lemon:lexicalForm on the lemon:LexicalEntry class. However, all datasets had the appropriate number of lemon:canonicalForm properties, which is a sub-property of lemon:lexicalForm and invalidates these errors. This constraint of RDFUnit, stems from the fact that transitive sub-property checking is not implemented at the time of writing. Except from the DBpedia Wiktionary dataset, all other lemon datasets had many reports of a lemon:LemonEntry without a label.

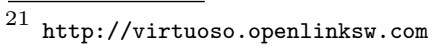


The DBpedia Wiktionary dataset had only five failed test cases. With an addition to the previous three, the dataset returned $163 \mathrm{~K}$ violations due to the disjointness of the lemon:LexicalEntry class with the lemon:LexicalSense class constraint and $3.5 \mathrm{M}$ violations of missing a required lemon:lexicalForm property in a lemon:LexicalEntry. The same query returned $270 \mathrm{~K}$ errors in the QHL dataset.

The Uby Wiktionaries had many failed test cases with a very low (less than 10) number of violations except from owl:minCardinality of one in lemon:Form class for the lemon:representation property. This test case returned $430 \mathrm{~K}$ errors on the English version and 200K errors on the German. Wordnet also failed this test case with $130 \mathrm{~K}$ violations. Finally, other high in number of violation test cases are found in the QHL dataset and regard incorrect domain $(30 \mathrm{~K})$ and range $(68 \mathrm{~K})$ of lemon: entry and wrong range of lemon:sense $(67 \mathrm{~K})$.

The most common test case that failed in all NIF datasets is the incorrect datatype of nif:beginIndex and nif:endIndex. Both properties are defined as xsd:nonNegativeInteger but were used as string Literals. This is due to a recent change of the NIF specification but also showcases the usefulness of our methodology for data evolution. The correct datatype of nif : beginIndex and nif : endIndex are also the reason for the NIF test cases that returned an error. In these cases, substrings based on these properties were calculated on the query (cf. Section 4.2 and non-numeric values did not allow a proper SPARQL query evaluation. This case also expresses the need for chained test cases execution (TestCaseDependency in cf. Section 3). The existence of a nif : beginIndex and nif : endIndex in a nif : CString also return violation in spotlight (68) kore50 (51) and Wikilinks $(21 \mathrm{~K})$ datasets. Finally $21 \mathrm{~K}$ objects in a nif : wasConvertedFrom relation did not have nif:String as range.

A direct comparison or our results with the results of the implemented validators cannot be provided in a consistent way. The NIF validator contained only 10 test cases while our approach had a total of 96 test cases. The lemon validator on the other hand could not finish after 48 hours for the DBpedia Wiktionay dataset and resulted in a multitude of non-RDF logging messages that were hard to filter and aggregate.

\section{Related Work}

There exist several approaches for assessing the quality of Linked Data. They can be broadly classified into (i) automated (e.g. [8]), (ii) semi-automated (e.g. [6]) or (iii) manual (e.g. 314]) methodologies. These approaches are useful at the process level wherein they introduce systematic methodologies to assess the quality of a dataset. However, the drawbacks include a considerable amount of user involvement, inability to produce interpretable results, or not allowing a user the freedom to choose the input dataset. In [11] errors occurring while publishing RDF data along were detected with a description of effects and means to improve the quality of structured data on the web. In a recent study, 4 million $\mathrm{RDF} / \mathrm{XML}$ documents were analysed which provided insights into the level of 
conformance these documents had in accordance to the Linked Data guidelines. On the one hand, these efforts contributed towards assessing a vast amount of Web or RDF/XML data, however, most of the analysis was performed automatically, therefore overlooking the problems arising due to contextual discrepancies.

The approach described in [7] advocates the use of SPARQL and SPIN for RDF data quality assessment and shares some similarity with our methodology. However, a domain expert is required for the instantiation of test patterns. SPARQL Inferencing Notation (SPIN) ${ }^{22}$ is a W3C submission aiming at representing rules and constraints on Semantic Web models. SPIN also allows users to define SPARQL functions and reuse SPARQL queries. The difference between SPIN and our pattern syntax, is that SPIN functions would not fully support our Pattern Bindings. SPIN function arguments must have specific constraints on the argument datatype or argument class and do not support operators, e.g. ' $=$ ', '>', '!', '+', '*', or property paths ${ }^{23}$ However, our approach is still compatible with SPIN when allowing to initialise templates with specific sets of applicable operators. In that case, however, the number of templates increases. Due to this restrictions, with SPIN we can define fewer but more general constraints. One of the advantages of converting our templates to SPIN is that the structure of the SPARQL query itself can be stored directly in RDF, which is, however, very complex and difficult to manage. From the efforts related to SPIN, we re-used their existing data quality patterns and ontologies for error types.

Pellet Integrity Constraint Validator [17](ICV) ${ }^{24}$ translates OWL integrity constraints into SPARQL queries. Similar to our approach, the execution of those SPARQL queries indicates violations. An implication of the integrity constraint semantics of Pellet ICV is that a partial unique names assumption (all resources are considered to be different unless equality is explicitly stated) and a closed world assumption is in effect. We use the same strategy as part of our methodology, but go beyond it by allowing users to directly (re-)use DQTPs not necessarily encoded in OWL.

\section{Conclusion and Future Work}

In this article, we extended a previously introduced methodology for test-driven quality assessment. In particular, a data engineering ontology was described in detail. We applied the RDFUnit Suite implementing this methodology to the NLP domain, an area in which RDF usage is currently rising and there is a need for quality assessment. In particular, we devised 277 test cases for NLP datasets using the Lemon and NIF vocabularies.

In future work, we aim to extend the test cases to more NLP ontologies, such as MARL, NERD and ITSRDF. We also plan to further increase the scope of the framework, e.g. for the recently changed namespaces of NIF and lemon deprecation warnings should be produced. Another extension is the modeling of

22 http://www.w3.org/Submission/spin-overview/

23 http://www.w3.org/TR/2010/WD-sparql11-property-paths-20100126/

24 http://clarkparsia.com/pellet/icv/ 
dependencies between test cases, which is currently done manually and could be automated. Furthermore, we also want to apply our methods on services: Usually, semantically enriched NLP services use text as input and return annotations in RDF, which could then be verified by RDFUnit to validate their output.

Acknowledgments. We would like to thank John McCrae for his support regarding lemon. This work was supported by grants from the EU's 7th Framework Programme provided for the projects LIDER (GA no. 610782), LOD2 (GA no. 257943) and GeoKnow (GA no. 318159).

\section{References}

1. R. Angles and C. Gutierrez. The expressive power of sparql. In The Semantic Web-ISWC 2008, pages 114-129. Springer, 2008.

2. K. Belhajjame, J. Cheney, D. Corsar, D. Garijo, S. Soiland-Reyes, S. Zednik, and J. Zhao. Prov-o: The prov ontology. Technical report, 2013.

3. C. Bizer and R. Cyganiak. Quality-driven information filtering using the WIQA policy framework. Web Semantics, 7(1):1 - 10, Jan 2009.

4. L. Bühmann and J. Lehmann. Pattern based knowledge base enrichment. In ISWC, 2013.

5. J. Eckle-Kohler, J. P. McCrae, and C. Chiarcos. lemonuby - a large, interlinked, syntactically-rich resource for ontologies. Submitted to the Semantic Web Journal.

6. A. Flemming. Quality characteristics of linked data publishing datasources. Master's thesis, Humboldt-Universität of Berlin, 2010.

7. C. Fürber and M. Hepp. Using sparql and spin for data quality management on the semantic web. In W. Abramowicz and R. Tolksdorf, editors, BIS, volume 47 of Lecture Notes in Business Information Processing, pages 35-46. Springer, 2010.

8. C. Guéret, P. T. Groth, C. Stadler, and J. Lehmann. Assessing linked data mappings using network measures. In $E S W C, 2012$.

9. S. Hellmann, J. Brekle, and S. Auer. Leveraging the crowdsourcing of lexical resources for bootstrapping a linguistic data cloud. In JIST, 2012.

10. S. Hellmann, J. Lehmann, S. Auer, and M. Brümmer. Integrating nlp using linked data. In $I S W C, 2013$.

11. A. Hogan, A. Harth, A. Passant, S. Decker, and A. Polleres. Weaving the pedantic web. In $L D O W, 2010$.

12. D. Kontokostas, P. Westphal, S. Auer, S. Hellmann, J. Lehmann, and R. Cornelissen. Test-driven evaluation of linked data quality. In $W W W, 2014$ (to appear).

13. J. McCrae, G. Aguado-de Cea, P. Buitelaar, P. Cimiano, T. Declerck, A. Gmez-Prez, J. Gracia, L. Hollink, E. Montiel-Ponsoda, D. Spohr, and T. Wunner. Interchanging lexical resources on the semantic web. LRE, 46(4):701-719, 2012.

14. P. N. Mendes, H. Mühleisen, and C. Bizer. Sieve: linked data quality assessment and fusion. In EDBT/ICDT Workshops, pages 116-123. ACM, 2012.

15. S. Moran and M. Brümmer. Lemon-aid: using lemon to aid quantitative historical linguistic analysis. In $L D L, 2013$.

16. M. Röder, R. Usbeck, S. Hellmann, D. Gerber, and A. Both. N3 - a collection of datasets for named entity recognition and disambiguation in the nlp interchange format. In $L R E C, 2014$.

17. E. Sirin and J. Tao. Towards integrity constraints in owl. In Proceedings of the Workshop on OWL: Experiences and Directions, OWLED, 2009.

18. N. Steinmetz, M. Knuth, and H. Sack. Statistical Analyses of Named Entity Disambiguation Benchmarks. In NLP and DBpedia WS @ ISWC, 2013. 